\title{
An HIV-positive Case of Obstructive Jaundice Caused by Immune Reconstitution Inflammatory Syndrome of Tuberculous Lymphadenitis Successfully Treated with Corticosteroids
}

\author{
Naoaki Watanabe ${ }^{1}$, Ryota Sato ${ }^{1}$, Hideaki Nagai ${ }^{1}$, Hirotoshi Matsui ${ }^{1}$, Akira Yamane ${ }^{1}$, \\ Masahiro Kawashima ${ }^{1}$, Junko Suzuki ${ }^{1}$, Hiroyuki Tashimo ${ }^{1}$, Nobuharu Ohshima ${ }^{1}$, \\ Kimihiko Masuda ${ }^{1}$, Atsuhisa Tamura ${ }^{1}$, Shinobu Akagawa ${ }^{1}$, Akira Hebisawa ${ }^{2}$ and Ken Ohta ${ }^{1}$
}

\begin{abstract}
:
A 60-year-old man was admitted to our hospital because of a persistent fever with enlargement of multiple lymph nodes in the mediastinum and around the pancreatic head. He was diagnosed with tuberculosis and human immunodeficiency virus infection. We started antiretroviral therapy three weeks after the initiation of anti-tuberculous therapy. Two weeks later, jaundice appeared with dilatation of the biliary tract due to further enlargement of the lymph nodes, which seemed to be immune reconstitution inflammatory syndrome (IRIS). The administration of corticosteroids resolved the obstructive jaundice without surgical treatment or endoscopic drainage. Obstructive jaundice caused by IRIS should first be treated with corticosteroids before invasive treatment.
\end{abstract}

Key words: HIV, immune reconstitution inflammatory syndrome, obstructive jaundice, tuberculous lymphadenitis

(Intern Med 56: 2661-2666, 2017)

(DOI: 10.2169/internalmedicine.8713-16)

\section{Introduction}

Immune reconstitution inflammatory syndrome (IRIS) is widely recognized as the paradoxical deterioration or emersion of opportunistic infections in patients infected with human immunodeficiency virus (HIV) receiving antiretroviral therapy (ART) accompanied by the recovery of host immunity (1-4). Though some obstructive jaundice cases caused by abdominal tuberculous lymphadenitis have been reported (5-10), there have been no reports of cases caused by the worsening of lymph node swelling due to IRIS. Endoscopic drainage or surgical treatment have been needed in most patients in addition to anti-tuberculous therapy (5-9). We herein report a case of obstructive jaundice caused by IRIS of tuberculous lymphadenitis successfully treated with corticosteroids.

\section{Case Report}

A 60-year-old man was admitted to our hospital because of a persistent fever lasting for 3 months. Chest X-ray showed no abnormalities in either lung fields. Computed tomography (CT) showed multiple low-density masses with an enhanced rim in the mediastinum and around the pancreatic head without lung involvement (Fig. 1a-d). The diagnosis of tuberculosis was made because acid-fast bacilli were detected in the gastric juice. A nucleic acid amplification test of the gastric juice for Mycobacterium tuberculosis was positive. He was also diagnosed with acquired immunodeficiency syndrome (AIDS) because the serologic test for HIV was positive. His medical history was significant, with histo-

${ }^{1}$ Center for Pulmonary Diseases, National Hospital Organization Tokyo National Hospital, Japan and ${ }^{2}$ Department of Clinical Research, National Hospital Organization Tokyo National Hospital, Japan

Received: December 13, 2016; Accepted: February 15, 2017; Advance Publication by J-STAGE: September 6, 2017

Correspondence to Dr. Ryota Sato, satour@tokyo-hosp.jp 

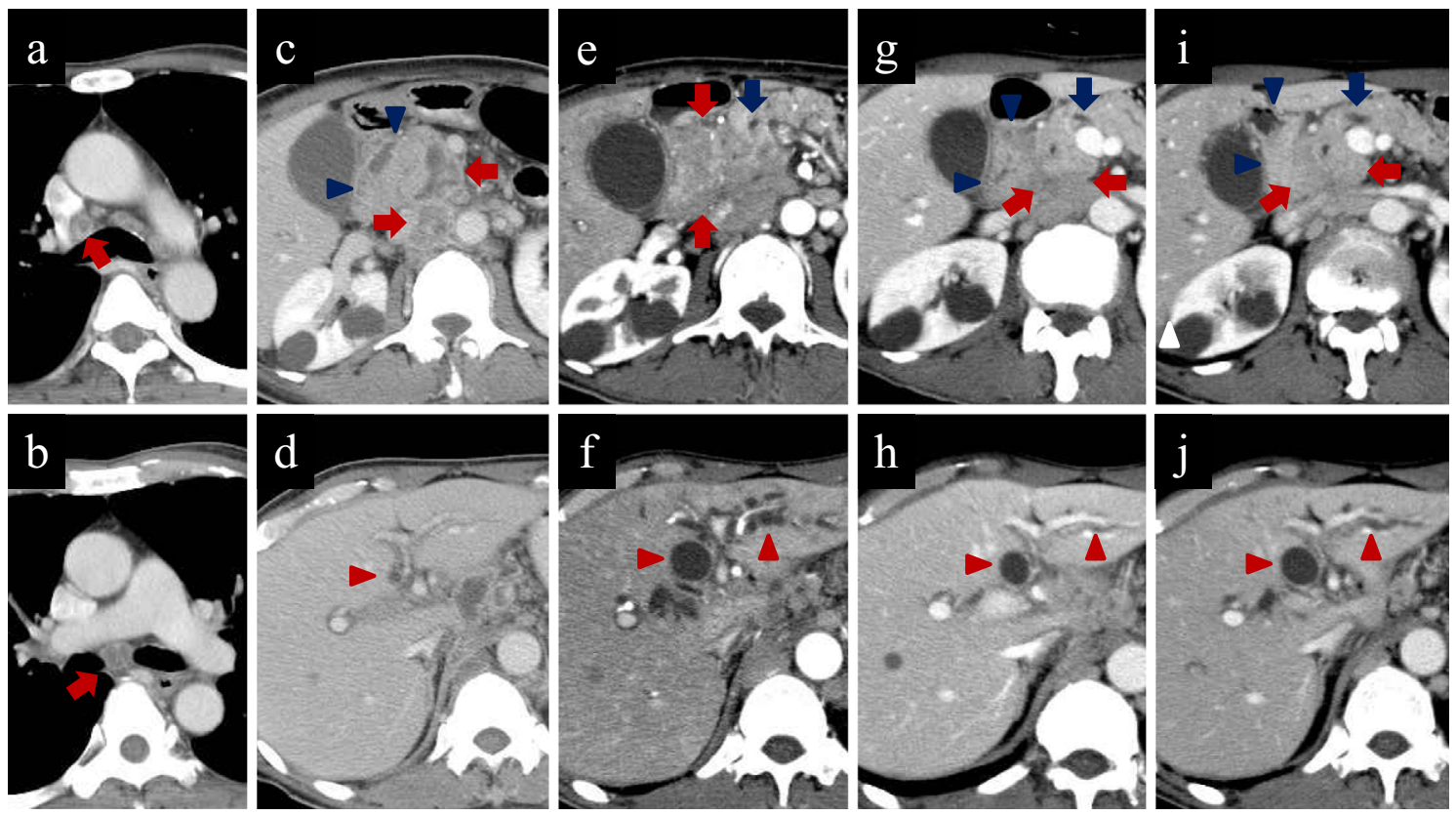

Figure 1. CT scans on admission showed multiple enlarged lymph nodes with an enhanced rim in the mediastinum and around the pancreatic head (a-d). After the initiation of ART, the biliary tracts and pancreatic duct were dilated due to worsening of the lymph node swelling around the pancreatic head $(e, f)$. The lymph nodes were reduced in volume with the administration of corticosteroids (g). The intrahepatic bile ducts and the pancreatic duct were normalized (h). After the withdrawal of corticosteroids, the dilatation of the common bile duct and intrahepatic bile ducts deteriorated, accompanied by slight worsening of the lymph node swelling $(i, j)$. The red arrow, red arrow head, blue arrow, and blue arrow head indicate the lymph node, biliary tract, pancreatic duct, and duodenum, respectively.

ries of herpes zoster at 53 years of age and amoebic enteritis at 58 years of age. There were no remarkable abnormalities in his physical findings. Laboratory examinations revealed a white blood cell count of $3,600 / \mu \mathrm{L}$, total bilirubin (T.Bil) $0.6 \mathrm{mg} / \mathrm{dL}$, aspartate aminotransferase (AST) $38 \mathrm{IU} / \mathrm{L}$, alanine aminotransferase (ALT) 23 IU/L, alkaline phosphate (ALP) 287 IU/L, gamma glutamyl transferase ( $\gamma \mathrm{GT}$ ) $100 \mathrm{IU} /$ L, CD4-positive lymphocyte counts $39 / \mu \mathrm{L}$, and HIV-RNA $1.72 \times 10^{5}$ copies $/ \mathrm{mL}$.

After the diagnosis of tuberculosis, we administered isoniazid (INH) $300 \mathrm{mg} /$ day, rifampicin (RFP) $450 \mathrm{mg} /$ day, ethambutol (EB) $750 \mathrm{mg} /$ day, and pyrazinamide (PZA) $1,200 \mathrm{mg} / \mathrm{day}$. One week after the initiation of antituberculous drugs, AST and ALT increased to $122 \mathrm{IU} / \mathrm{L}$ and $62 \mathrm{IU} / \mathrm{L}$, respectively, but normalized after the discontinuation of PZA. Three weeks after the initiation of antituberculous drugs, we started ART with tenofovir $300 \mathrm{mg} /$ day, emtricitabin $200 \mathrm{mg} /$ day, and raltegravir 1,600 mg/day. Subsequently, he underwent gastroduodenoscopy because of appetite loss and epigastric pain and was discovered to have duodenal stenosis with mucosal edema (Fig. 2a). The granulomatous lesion and acid-fast bacilli in the biopsy specimen of duodenum led to the additional diagnosis of duodenal tuberculosis (Fig. 3). Two weeks after the initiation of ART, he presented with jaundice with T.Bil $5.26 \mathrm{mg} / \mathrm{dL}$ and direct bilirubin $3.91 \mathrm{mg} / \mathrm{dL}$. Other laboratory findings were serum

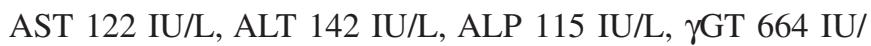
$\mathrm{L}$, and amylase $476 \mathrm{IU} / \mathrm{L}$ at that time. Enhanced CT revealed dilatations of the intrahepatic bile ducts, the common bile duct, and the pancreatic duct due to worsening of lymph node swelling around the pancreatic head (Fig. 1e and f). Given his increased number of CD4-positive lymphocytes $(82 / \mu \mathrm{L})$ and the decreased HIV-RNA level $\left(2.68 \times 10^{2}\right.$ copies/mL), we considered the obstructive jaundice to be a consequence of IRIS. We subsequently switched his anti-tuberculous drugs to INH, EB, and levofloxacin, and the jaundice disappeared; the elevated liver enzymes and pancreatic enzyme also decreased to the normal range after the initiation of $60 \mathrm{mg} / \mathrm{day}$ of oral prednisolone. At almost the same time, the swelling of the lymph nodes and dilatation of the bile ducts and pancreatic duct were improved. (Fig. 1g and h). Improvement of the duodenal stenosis with mucosal edema was also confirmed (Fig. 2b). The dosage of the corticosteroids was reduced and then discontinued within a month, but the biliary and pancreatic enzyme levels in-

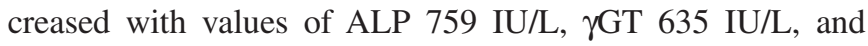
amylase $250 \mathrm{IU} / \mathrm{L}$ at 2 weeks after the withdrawal of corticosteroids, and dilatations of the common bile duct and intrahepatic bile ducts recurred (Fig. 1i and $\mathrm{j}$ ).

We administered oral prednisolone $30 \mathrm{mg} /$ day again and tapered the administration over the next 9 months. Disappearance of the enlarged lymph nodes was confirmed by CT 


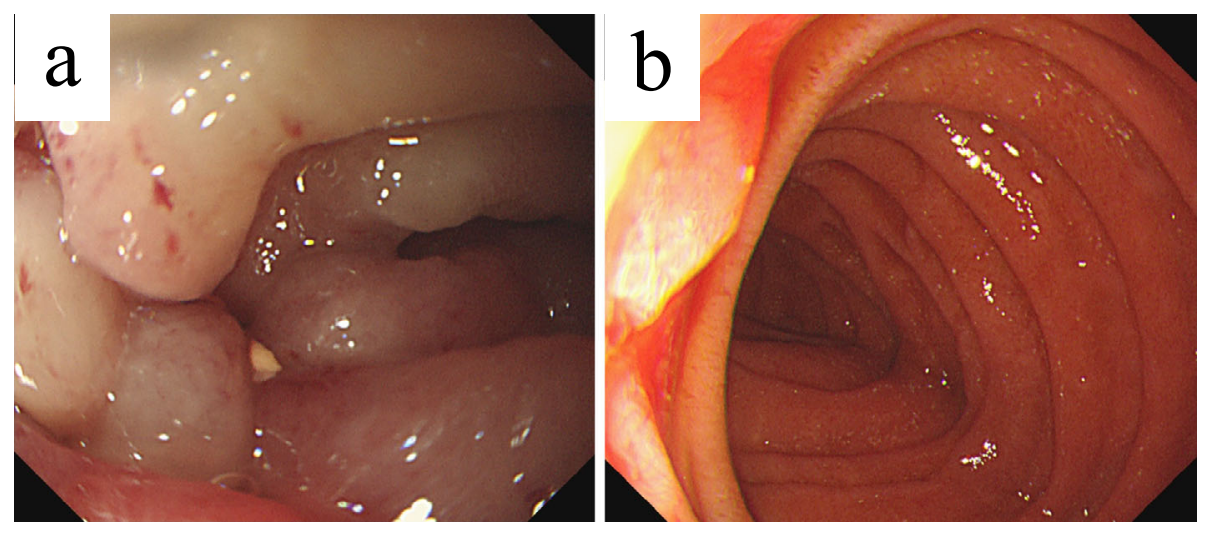

Figure 2. A gastroduodenal endoscopic examination. Before the administration of corticosteroids, showing duodenal stenosis with mucosal edema (a). After treatment with corticosteroids, mucosal edema was improved, and the ampulla of Vater was visible (b).
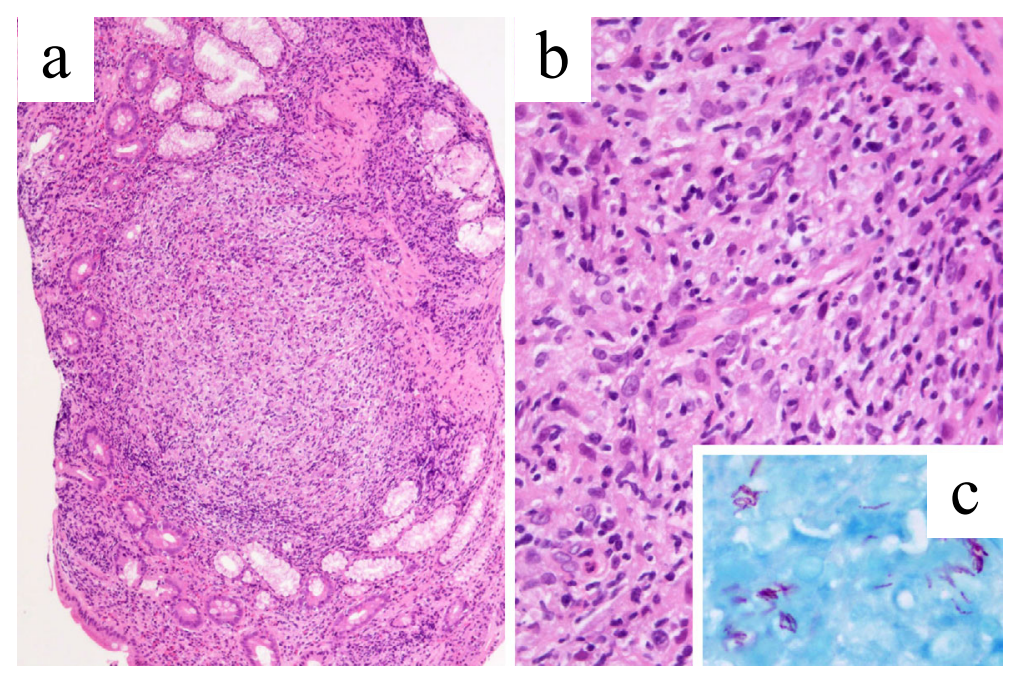

Figure 3. Histopathology of the duodenal biopsy showing granulomatous lesion consisting of macrophages. [a: Hematoxylin and Eosin $(H \& E)$ staining, $\times 100$, b: H\&E staining, $\times 400]$. Acid-fast bacilli were detected with a Ziehl-Neelsen stain $(c: \times 1,000)$.

seven months after retreatment with corticosteroids, and no relapse of obstructive jaundice was noted subsequently. Regarding the treatment of tuberculosis, we initially administered INH, RFP, and EB for 2 months followed by INH and RFP for 10 months because he was immunocompromised. The clinical course of the treatment and examination data are shown in Fig. 4.

\section{Discussion}

Obstructive jaundice is reportedly caused by abdominal tuberculous lymphadenitis obstructing biliary tracts around the hepatic portal region and/or around the pancreatic head (5-10). In the present case, lymph node swelling around the pancreatic head worsened after the initiation of ART, thereby inducing pancreatic and bile duct obstruction. In addition, duodenal tuberculosis can obstruct the ampulla of Vater and promote obstructive jaundice. In an analysis of 23 gastroduodenal tuberculosis cases, 2 patients with pe- riampullary lesion had jaundice (11).

It is difficult to diagnose abdominal tuberculous lymphadenitis without pulmonary lesions because the detection of tuberculous bacilli from the abdominal lesions can occur due to invasive procedures, laparotomy or laparoscopy $(6,7,9)$. However, the usefulness of endoscopic ultrasound-guided fine-needle aspiration for such a diagnosis was reported recently $(8,10)$. In the present case, the detections of tuberculous bacilli in the gastric juice and duodenal epithelia, in addition to the observation of enlarged, lowdensity lymph nodes with rim enhancement, led to a diagnosis without a biopsy of the lymph nodes.

In HIV-infected patients with advanced immunodeficiency, opportunistic infections frequently emerge or deteriorate paradoxically after the initiation of ART. This phenomenon, called IRIS, is caused by excessive inflammation in response to pathogens in the course of the recovery of host immunity (1-4). Mycobacterium tuberculosis is one of the most common pathogens causing IRIS, with an incidence rate of 


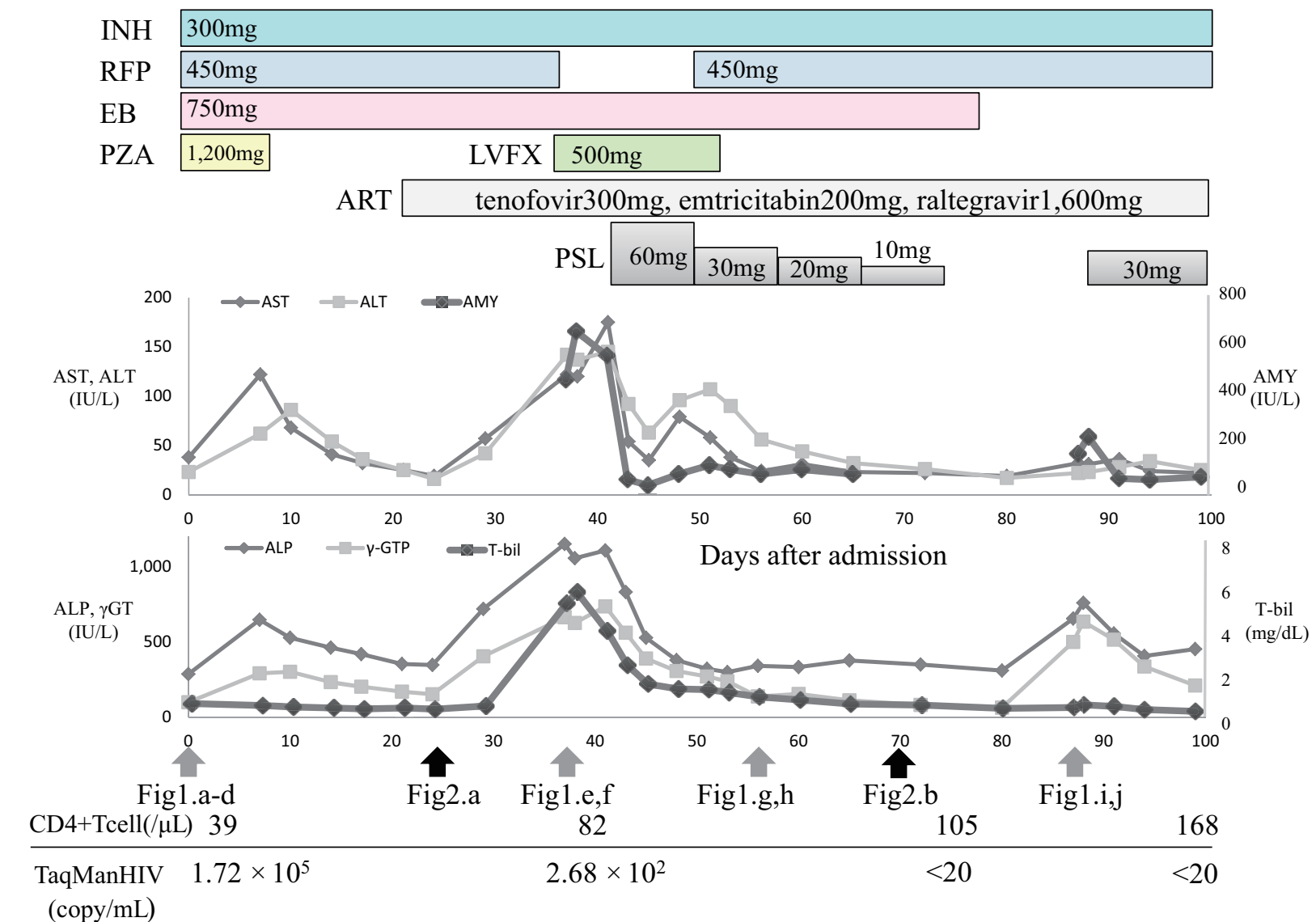

Figure 4. Time course of the laboratory data, treatment, and examinations. Three weeks after the initiation of anti-tuberculous drugs, ART was started with tenofovir, emtricitabin, and raltegravir. Two weeks after the initiation of ART, jaundice appeared, with elevated hepatobiliary and pancreatic enzymes, which improved by the administration of corticosteroids. The biliary and pancreatic enzymes increased again after the withdrawal of corticosteroids, and corticosteroid administration was restarted to resolve the relapsed IRIS. INH: isoniazid, RFP: rifampicin, EB: ethambutol, PZA: pyrazinamide, LVFX: levofloxacin, ART: antiretroviral therapy, PSL: prednisolone

\section{$15.7 \%$ for active tuberculosis (2).}

Shelburne et al. (3) suggested four diagnostic criteria of IRIS as follows: [1] HIV infection, [2] receiving ART, a decrease in the HIV-RNA level and an increase in the number of CD4-positive cells, [3] clinical symptoms consistent with inflammatory processes, and [4] a clinical course not consistent with the expected course of previously or newly diagnosed opportunistic infections. In the present case, further swelling of the lymph nodes developed with increasing CD4-positive lymphocyte counts and a decreasing HIV-RNA burden despite effective anti-tuberculous therapy, suggesting IRIS according to Shelburne's criteria.

French et al. (12) reported that the risk factors of IRIS were a low CD4-positive lymphocyte count and a high HIVRNA burden at the initiation of ART. The risk for IRIS might have been high in this patient because his CD4positive lymphocyte count was $39 / \mu \mathrm{L}$ and his HIV-RNA burden was $1.72 \times 10^{5}$ copies $/ \mathrm{mL}$ at the initiation of ART. In active tuberculosis cases, a short interval from the initiation of anti-tuberculous therapy to ART was reported as a risk factor for IRIS (13-15). However, ART is recommended to be started within the first 2 weeks of the initiation of tuberculosis treatment for those with CD4 cell counts $<50$ / $\mu \mathrm{L}$ (16), so we stared ART as soon as possible after the improvement of liver injury due to anti-tuberculous therapy.

In general, the therapeutic strategy for IRIS is to keep treating the primary disease and to control excessive inflammation. In severe cases of IRIS, non-steroidal antiinflammatory drugs or corticosteroids are recommended (17). Although we tapered the corticosteroid administration within a month to avoid immunosuppression, the obstruction of the bile duct and pancreatic duct developed again. Readministration of corticosteroids for more than nine months was needed to avoid subsequent relapse.

We searched the literature between 1996 and 2016 using PubMed and found 17 cases of obstructive jaundice caused by abdominal tuberculous lymphadenitis ( Table) (5-10, 18-27). Nine underwent surgery to resolve obstructive jaundice, and five underwent percutaneous or endoscopic drainage in addition to anti-tuberculous drugs. In only three cases, the jaundice was improved without invasive treatment. Lee et al. (5) reported a case of non-HIV ob- 
Table. Seventeen Obstructive Jaundice Cases Induced by Tuberculous Lymphadenitis.

\begin{tabular}{cccccl}
\hline Reference No. & Sex/Age & HIV & T.Bil (mg/dL) & Diagnostic method & Treatment except medications \\
\hline$(7)$ & F/56 & - & 4.6 & laparotomy & choledochojejunostomy \\
$(18)$ & M/20 & N.M. & 17.4 & laparotomy & PTBD, choledochojejunostomy \\
$(18)$ & M/34 & N.M. & 7.7 & laparoscopy & PTBD \\
$(19)$ & F/68 & - & 4.6 & laparotomy & pancreatoduodenectomy \\
$(20)$ & M/32 & N.M. & 16.9 & laparotomy & cholecystectomy, T-tube drainage \\
$(21)$ & F/45 & - & 4.8 & laparotomy & pancreatoduodenectomy \\
$(22)$ & M/31 & N.M. & 8.7 & laparotomy & resection of lymph nodes \\
$(23)$ & F/42 & - & 8.4 & superficial LN biopsy & none \\
$(6)$ & M/29 & N.M. & 9.5 & laparotomy & choledochojejunostomy \\
$(5)$ & M/23 & - & 2.7 & colonoscopy & ERBD with a stent \\
$(10)$ & F/49 & N.M. & 2.5 & EUS-FNA & none \\
$(9)$ & M/30 & N.M. & 21.1 & laparotomy & PTBD, hepaticojejunostomy \\
$(24)$ & F/49 & N.M. & 3.9 & laparotomy & choledochoduodenostomy \\
$(25)$ & M/29 & N.M. & 4.2 & laparotomy & ERBD with a stent \\
$(26)$ & M/17 & N.M. & 1.6 & EUS-FNA & none \\
$(8)$ & F/33 & - & 5.6 & EUS-FNA & ERBD with a stent \\
$(27)$ & M/20 & N.M. & $1.2(※)$ & EUS-FNA & ERBD with a stent \\
\hline
\end{tabular}

HIV: human immunodeficiency virus, T.Bil: total bilirubin, F: female, M: male, N.M.: not mentioned, LN: lymph node, EUSFNA: endoscopic ultrasound-guided fine-needle aspiration, PTBD: percutaneous transhepatic biliary drainage, ERBD: endoscopic retrograde biliary drainage, $(※)$ : T.Bil after drainage

structive jaundice that was caused by a paradoxical reaction after the initiation of anti-tuberculous therapy. A paradoxical reaction to anti-tuberculous therapy is also thought to be associated with an excessive inflammatory reaction similar to IRIS (28). In those authors' report, steroid administration was insufficient, and endoscopic drainage was required to resolve the patient's jaundice.

This is the first report of obstructive jaundice caused by IRIS of tuberculous lymphadenitis. Though most cases of obstructive jaundice caused by tuberculous lymphadenitis have required surgical treatment or endoscopic drainage, the present case was successfully treated with corticosteroids. Obstructive jaundice caused by IRIS should be first treated with corticosteroids before invasive treatment is applied.

The authors state that they have no Conflict of Interest (COI).

\section{References}

1. Lawn SD, Meintjes G. Pathogenesis and prevention of immune reconstitution disease during antiretroviral therapy. Expert Rev Anti Infect Ther 9: 415-430, 2011.

2. Müller M, Wandel S, Colebunders R, et al. Immune reconstitution inflammatory syndrome in patients starting antiretroviral therapy for HIV infection: a systematic review and meta-analysis. Lancet Infect Dis 10: 251-261, 2010.

3. Shelburne SA, Montes M, Hamill RJ. Immune reconstitution inflammatory syndrome: more answers, more questions. J Antimicrob Chemother 57: 167-170, 2006.

4. French MA, Lenzo N, John M, et al. Immune restoration disease after the treatment of immunodeficient HIV-infected patients with highly active antiretroviral therapy. HIV Med 1: 107-115, 2000.

5. Lee YJ, Jung SH, Hyun WJ, et al. A case of obstructive jaundice caused by paradoxical reaction during antituberculous chemotherapy for abdominal tuberculosis. Gut Liver 3: 338-342, 2009.
6. Colovic R, Grubor N, Jesic R, et al. Tuberculous lymphadenitis as a cause of obstructive jaundice: a case report and literature review. World J Gastroenterol 14: 3098-3100, 2008.

7. Caroli-Bosc FX, Conio M, Maes B, Chevallier P, Hastier P, Delmont JP. Abdominal tuberculosis involving hepatic hilar lymph nodes. A cause of portal vein thrombosis and portal hypertension. J Clin Gastroenterol 25: 541-543, 1997.

8. Furuhashi H, Abe H, Yoshizawa K, et al. A case of peripancreatic tuberculous lymphadenitis diagnosed by endoscopic ultrasoundguided fine-needle aspiration. Clin J Gastroenterol 7: 68-73, 2014.

9. Baik SJ, Yoo K, Kim TH, Moon IH, Cho MS. A case of obstructive jaundice caused by tuberculous lymphadenitis: a literature review. Clin Mol Hepatol 20: 208-213, 2014.

10. Macías-Garcia F, Iglesias-García J, Abdulkader I, Lariño-Noia J, Forteza-Vila J, Dominguez-Muñoz JE. Tuberculous lymph node at the porta hepatis: diagnosis by EUS-guided FNA. Gastrointest Endosc 74: 437-439, 2011.

11. Rao YG, Pande GK, Sahni P, Chattopadhyay TK. Gastroduodenal tuberculosis management guidelines, based on a large experience and a review of the literature. Can J Surg 47: 364-368, 2004.

12. French MA, Lenzo N, John M, et al. Immune restoration disease after the treatment of immunodeficient HIV-infected patients with highly active antiretroviral therapy. HIV Med 1: 107-115, 2000.

13. Abdool Karim SS, Naidoo K, Grobler A, et al. Integration of antiretroviral therapy with tuberculosis treatment. N Engl J Med 365 : 1492-1501, 2011.

14. Blanc FX, Sok T, Laureillard D, et al. Earlier versus later start of antiretroviral therapy in HIV-infected adults with tuberculosis. $\mathrm{N}$ Engl J Med 365: 1471-1481, 2011.

15. Havlir DV, Kendall MA, Ive $P$, et al. Timing of antiretroviral therapy for HIV-1 infection and tuberculosis. $\mathrm{N}$ Engl $\mathrm{J}$ Med 365: 1482-1491, 2011.

16. Günthard HF, Saag MS, Benson CA, et al. Antiretroviral drugs for treatment and prevention of HIV infection in adults: 2016 recommendations of the international antiviral society-USA Panel. JAMA 316: 191-210, 2016.

17. Murthy AR, Marulappa R, Hegde U, Kappadi D, Ambikathanaya UK, Nair P. Treatment guidelines and prognosis of immune reconstitution inflammatory syndrome patients: a review. J Int Oral 
Health 7: 92-95, 2015.

18. Poon RT, Lo CM, Fan ST. Diagnosis and management of biliary obstruction due to periportal tuberculous adenitis. Hepatogastroenterology 48: 1585-1587, 2001.

19. Turan M, Sen M, Koyuncu A, Aydin C, Elaldi N, Arici S. Pancreatic pseudotumor due to peripancreatic tuberculous lymphadenitis. Pancreatology 2: 561-564, 2002.

20. Obama K, Kanai M, Taki Y, Nakamoto Y, Takabayashi A. Tuberculous lymphadenitis as a cause of obstructive jaundice: report of a case. Surg Today 33: 229-231, 2003.

21. Sen M, Turan M, Karadayi K, Aslan M, Elagoz S. Peripancreatic tuberculous lymphadenitis mimicking carcinoma: report of a case. Acta Chir Belg 104: 338-340, 2004.

22. Peyré CG, Wakim M, Mateo R, et al. Unusual cases of jaundice secondary to non-neoplastic bile duct obstruction. Am Surg 70: 620-624, 2004.

23. Cherian JV, Somasundaram A, Ponnusamy RP, Venkataraman J. Peripancreatic tuberculous lymphadenopathy. An impostor posing diagnostic difficulty. JOP 8: 326-329, 2007.

24. Jeremic L, Stojanovic M, Radojkovic M, Zlatic A, Ignjatovic N, Jeremic S. Tuberculous lymphadenitis as a cause of obstructive jaundice. Chirurgia (Bucur) 108: 725-728, 2013.

25. Fernández Muinelo A, Salgado Vázquez M, Núñez Fernández S, Pardo Rojas P, Gómez Lorenzo FJ. Obstructive jaundice secondary to liver hilar lymph node tuberculosis. Cir Esp 91: 611-612, 2013.

26. Lee YJ, Hwang JY, Park SE, Kim YW, Lee JW. Abdominal tuberculosis with periportal lymph node involvement mimicking pancreatic malignancy in an immunocompetent adolescent. Pediatr Radiol 44: 1450-1453, 2014.

27. Kuriry H, Alenezi R, Alghamdi A, Swied AM. Isolated Retropancreatic Tuberculous Lymphadenitis Mimicking Carcinoma: A Diagnostic Challenge. Case Rep Gastrointest Med 2016: 7295496, 2016.

28. Bell LC, Breen R, Miller RF, Noursadeghi M, Lipman M. Paradoxical reactions and immune reconstitution inflammatory syndrome in tuberculosis. Int J Infect Dis 32: 39-45, 2015.

The Internal Medicine is an Open Access article distributed under the Creative Commons Attribution-NonCommercial-NoDerivatives 4.0 International License. To view the details of this license, please visit (https://creativecommons.org/licenses/ by-nc-nd/4.0/).

(C) 2017 The Japanese Society of Internal Medicine

Intern Med 56: 2661-2666, 2017 